\title{
Absorbers in the suburbs: HST views the local Ly $\alpha$ forest
}

\author{
John T. Stocke, J. M. Shull, S. V. Penton, \\ B. A. Keeney and J. L. Rosenberg
}

CASA, Univ. of Colorado, Boulder CO 80309-0389, USA email: stocke@casa.colorado.edu

\begin{abstract}
We describe recent discoveries of low column density $\left(\mathrm{N}_{\mathrm{HI}}=10^{12.5-16.0} \mathrm{~cm}^{-2}\right) \mathrm{H} \mathrm{I}$ Ly $\alpha$ absorbers made with the Hubble Space Telescope (HST) which have allowed us a first look at gas in local intergalactic space; i.e., between us and the "Great Wall". These absorbing clouds account for $29 \pm 4 \%$ of all baryons at $z=0$ and are, in general, only loosely related to individual galaxies. Owing to the proximity of these absorbers to the Earth, the 187 absorbers in our combined GHRS + STIS sample provide our best view of the relationship between Ly $\alpha$ absorbers and galaxies, voids, and supercluster filaments. While only a few of the very strongest absorbers in our sample appear associated with individual galaxies, the majority (78\%) are associated with large-scale filamentary structures of galaxies, while $22 \%$ are found in galaxy "voids". Amongst the highest column density absorbers (with $\mathrm{N}_{\mathrm{HI}} \approx 10^{16} \mathrm{~cm}^{-2}$ ) in our sample, we find a low-ionization, metal-bearing absorber associated with a post-starburst dwarf galaxy $70 \mathrm{~h}_{70}^{-1} \mathrm{kpc}$ away. The detailed properties of the absorber and the galaxy provide strong evidence that these two are causally related. We argue that many/most weak metal absorption systems could be due to outflowing "superwinds" from dwarf galaxies.
\end{abstract}

\section{The HST + STIS \& GHRS Ly $\alpha$ absorber sample}

Unlike virtually all other astronomical objects, Ly $\alpha$ absorbing "clouds" were first discovered at great distances $(z \geqslant 2)$ due to cosmological redshifts and the near-UV atmospheric cutoff. It has only been with the advent of the Hubble Space Telescope (HST) that nearby examples have been found and studied (Bahcall et al. 1991, 1993; Morris et al. 1991; and subsequent HST QSO Absorption Line Key Project papers by Jannuzi et al. 1998 and Weymann et al. 1998). While these absorbers are abundant enough to account for all baryons at $z \geqslant 2$, their still substantial numbers at $z=0$ imply that $\sim 30 \%$ of all baryons remain in these clouds locally (Penton et al. 2000a,b; 2002, 2004; Papers I - IV hereafter, and Davé et al. 1998, 2000). Thus, any account of the present-day distribution of baryons must include an accurate census of these clouds and their masses, as inferred from their column densities, ionized fractions and physical extents.

Surprisingly and luckily for local IGM research, the modest HST aperture is competitive with the $10 \mathrm{~m}$ aperture of the Keck Telescope (+HIRES spectrograph) in detecting Ly $\alpha$ absorbers because much brighter targets can be observed. Figure 1 shows an HST+STIS (Space Telescope Imaging Spectrograph) far-UV spectrum of the bright BL Lac Object PKS 2005-489, which detects Ly $\alpha$ absorbers as low in column density, $\mathrm{N}_{\mathrm{HI}} \geqslant 10^{12.5} \mathrm{~cm}^{-2}$, as the best Keck HIRES data (e.g., Hu et al. 1995), but these HST-discovered absorbers are within $20,000 \mathrm{~km} \mathrm{~s}^{-1}$ of the Earth. This allows us an unprecedented opportunity to search for faint galaxies that could be associated with these absorbers.

All results reported here are based on data toward 15 targets studied with the Goddard High Resolution Spectrograph (GHRS) (Papers I - III) and 16 targets observed using STIS (Paper IV). In the entire survey, we have detected $187 \mathrm{Ly} \alpha$ absorbers at $\geqslant 4 \sigma$ 


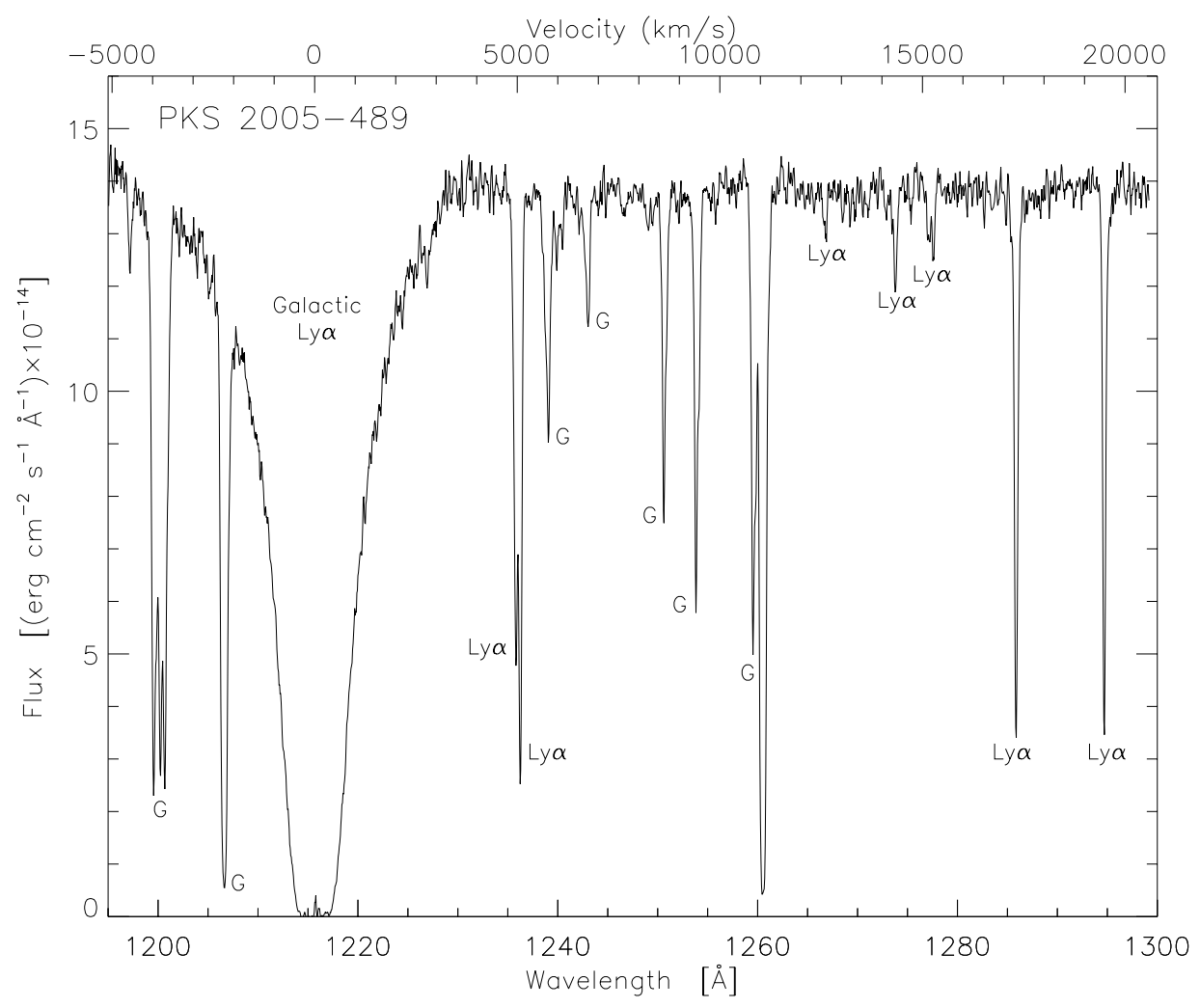

Figure 1. An HST/STIS medium resolution $\left(20 \mathrm{~km} \mathrm{~s}^{-1}\right)$ spectrum (Paper IV) of the bright BL Lac Object PKS 2005-489 illustrates the best quality data obtained for this project. The deep, broad absorption at $1216 \AA$ is the damped Ly $\alpha$ absorption due to the Milky Way. Other Galactic metal lines (S II, Si II, Mg II, N I, N V and Si III) are marked with a "G". The weakest Ly $\alpha$ absorbers have column densities, $\mathrm{N}_{\mathrm{HI}}=10^{12.5} \mathrm{~cm}^{-2}$. The heliocentric velocity scale at top is for the Ly $\alpha$ absorbers only.

significance over a total unobscured path length $\Delta z=1.16$. This yields $d N / d z \sim 300$ per unit redshift at $\mathrm{N}_{\mathrm{HI}} \geqslant 10^{12.5} \mathrm{~cm}^{-2}$ or one "cloud" every $15 h_{70}^{-1} \mathrm{Mpc}$. Using two different methods (Paper II and Schaye 2001) we estimate that the local, photoionized IGM contains $29 \pm 4 \%$ of all baryons at $z=0$ (see Paper IV for details). Since Ly $\alpha$ becomes extremely difficult to detect in our spectra at $\mathrm{T} \geqslant 150,000^{\circ} \mathrm{K}$, the baryon fraction above does not include much of the "warm-hot" medium predicted to account for another $~ 10$ $30 \%$ of all baryons (Cen et al. 1994; Davé et al. 2000).

For a subset of 79 absorbers in our sample which lie in regions of the local universe surveyed for galaxies down to at least $\mathrm{L}^{*}$ (based upon the CfA redshift survey catalog; Huchra et al. 1992, 2004 version), we find that $78 \%$ of all low column density absorbers lie in galaxy filaments and $22 \%$ lie in galaxy voids (nearest neighbor galaxy distances $\left.>3 \mathrm{~h}_{70}^{-1} \mathrm{Mpc}\right)$. The stronger absorbers $\left(\mathrm{N}_{H I} \geqslant 10^{13.1} \mathrm{~cm}^{-2}\right)$ have much smaller nearest galaxy neighbor distances (median distance $\approx 400 \mathrm{~h}_{70}^{-1} \mathrm{kpc}$ ) than the weaker absorbers (median distance $\approx 1 \mathrm{~h}_{70}^{-1} \mathrm{Mpc}$ ). And, while the weaker absorbers cluster only very weakly with galaxies, they are not randomly distributed in space ( $7 \sigma$ difference).

The two-point correlation function (TPCF; see Figure 2) for all "local" Ly $\alpha$ absorbers suggests a similar difference between the stronger and weaker absorbers. The full sample has a $7 \sigma$ excess at $\Delta \mathrm{v} \leqslant 250 \mathrm{~km} \mathrm{~s}^{-1}$, but this excess is due entirely to the stronger half 


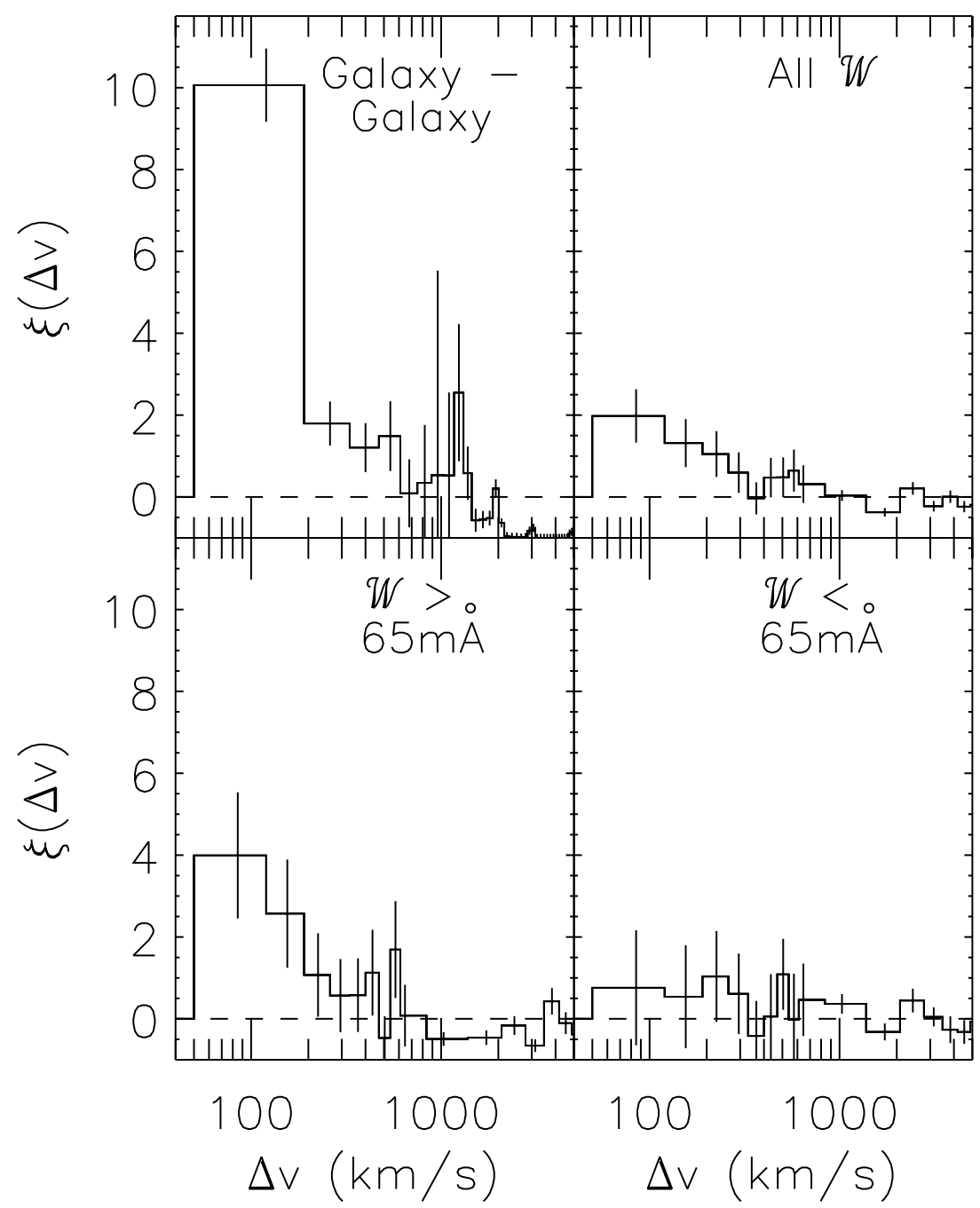

Figure 2. The two-point auto-correlation function amplitude (TPCF) for: galaxies in the CfA redshift survey region (upper left), our entire 187 Ly $\alpha$ sample (upper right), the stronger half $\left(\mathrm{N}_{H I} \geqslant 10^{13.1} \mathrm{~cm}^{-2}\right.$ ) of our Ly $\alpha$ sample (lower left) and the weaker half sample (lower right). The galaxy-galaxy TPCF shows both an extremely large excess power at $\Delta \mathrm{v} \leqslant 600 \mathrm{~km} \mathrm{~s}^{-1}$ due to galaxies within the same filament and a large deficit at larger $\Delta \mathrm{v}$ due to galaxy voids. Note that the stronger half-sample shows both the excess at small $\Delta \mathrm{v}$ and a hint of a deficit at larger $\Delta \mathrm{v}$, while the weaker half sample shows no evidence for clustering on any scale.

of the sample (compare upper left and lower left panels). The weaker absorbers show no evidence for clustering on any velocity scale, indicating a relatively random distribution in space (Paper IV). Thus, it is generally the weaker Ly $\alpha$ absorbers which are in galaxy voids. These differences are suggestive of two populations of absorbers (Sargent, 1978), the stronger of which are somehow associated with galaxies (and may contain metals) while the weaker may be pristine gas. 


\section{Ly $\alpha$ absorbers in galaxy voids}

When corrected for total path length surveyed, we find that $22 \%$ of all low column density Ly $\alpha$ absorbers are in galaxy voids, more than $3 \mathrm{~h}^{-1} \mathrm{Mpc}$ from the nearest galaxy. The number of $\operatorname{Ly} \alpha$ absorbers we find in voids constitutes $4.5 \pm 1.5 \%$ of the total baryon density, a number which agrees quite well with the total mass density in voids found in $\Lambda$ CDM simulations by Gottlöber et al. (2003). Additionally, we have conducted a deep optical search (McLin et al. 2002) and a sensitive H I 21cm search (Zamojski et al., in preparation) for galaxies and $\mathrm{H}$ I clouds near four very nearby void absorbers at $c z=2000-3000 \mathrm{~km} \mathrm{~s}^{-1}$. No optical galaxies were found down to very faint limits $\left(\mathrm{M}_{B} \leqslant\right.$ -14 within $250 \mathrm{~h}^{-1} \mathrm{kpc}$ of the absorber) but one $\mathrm{H}$ I emitting cloud (but no stars) was found $0.4 \mathrm{~h}^{-1} \mathrm{Mpc}$ away from one absorber. The detection of this one $\sim 10^{8} \mathrm{M}_{\odot}$ object is in approximate agreement with the number of dark matter halos expected in voids (Gottlöber et al. 2003).

Finally, we have searched the strongest Ly $\alpha$ absorbers in voids for metals, and have not detected any Si III, C IV or O VI absorptions down to levels of $[Z] \leqslant-2.0$ solar. While these limits are already significantly below what would be expected from currentepoch "superwinds" from galaxies, they do not eliminate the possibility of "Population III level" metallicities suggested to be present throughout the high- $z$ IGM (e.g., Cowie \& Songaila 1998; but see Simcoe, Sargent \& Rauch 2004).

\section{Weak metal-line absorbers and starburst winds}

Our low-column density Ly $\alpha$ sample provides a unique opportunity to study very nearby examples of Ly $\alpha$ forest absorbers. This is of particular importance for the weak metal-line systems because the origins of their metals is not well-understood. Two of the best examples of weak metal-line systems are found in the sightline pair 3C273/ RXJ $1230.8+0115$, which is separated by $0.91^{\circ}\left(350 \mathrm{~h}_{70}^{-1} \mathrm{kpc}\right.$ apart at $\left.c z=1600 \mathrm{~km} \mathrm{~s}^{-1}\right)$ on the sky. Both sightlines have Ly $\alpha$ absorbers at $\mathrm{N}_{H I} \approx 10^{16} \mathrm{~cm}^{-2}$ ) at similar redshift ( $c z=1586$ and $1666 \mathrm{~km} \mathrm{~s}^{-1}$ respectively) and metallicity (6\% and $10 \%$ solar; Tripp et al. 2002; Rosenberg et al. 2003). Recently, we (Stocke et al. 2004) have discovered a dwarf $\left(\mathrm{M}_{B}=-13.9\right)$, post starburst galaxy $70 h_{70}^{-1} \mathrm{kpc}$ away from the $3 \mathrm{C} 2731586 \mathrm{~km} \mathrm{~s} \mathrm{~s}^{-1}$ absorber mentioned above (see Figure 3). Not only do the absorber and galaxy redshifts match to within their combined errors but the absorber metallicity ( $6 \%$ solar) and the mean stellar metallicity of the galaxy $(\sim 10 \%)$ also approximately match. Further, the absorber has an overabundance of silicon to carbon indicative of recent supernova type II enrichment. The galaxy is a pure disk system whose optical spectrum has both strong Balmer and metal absorption lines, with no evidence for dust or gas (no emission lines and $\mathrm{M}_{H I} \leqslant 5 \times 10^{6} \mathrm{M}_{\odot}$; van Gorkom et al. 1993). From ratios of Lick absorption line indices, we estimate that the mean stellar age in this galaxy is $3.5 \pm 1.5$ Gyrs.

Taken together, this information provides a consistent picture in which a massive $(\geqslant$ $0.3 \mathrm{M}_{\odot} \mathrm{yr}^{-1}$ ) starburst $\sim 3$ Gyrs ago created enough supernovae to blow away the remaining gas from this galaxy into the IGM. Because the dwarf is quite low mass, this wind can easily escape from the galaxy and move $\sim 10^{8} \mathrm{M}_{\odot}$ of gas to $\sim 100 h_{70}^{-1} \mathrm{kpc}$ at the required $20-30 \mathrm{~km} \mathrm{~s}^{-1}$ to create the metal-line absorber that we see between us and 3C 273 (Stocke et al. 2004).

But because this is the nearest weak metal-line system known, many other similar absorbers could be due to starburst winds from dwarf galaxies, which are too faint to find and observe (e.g., at $z \approx 0.1$, a galaxy like this dwarf would have $\mathrm{m}_{B} \sim 24$ ). And because dwarf galaxies are so numerous $\left(1-3 \mathrm{Mpc}^{-3}\right)$, if each dwarf galaxy had at least 


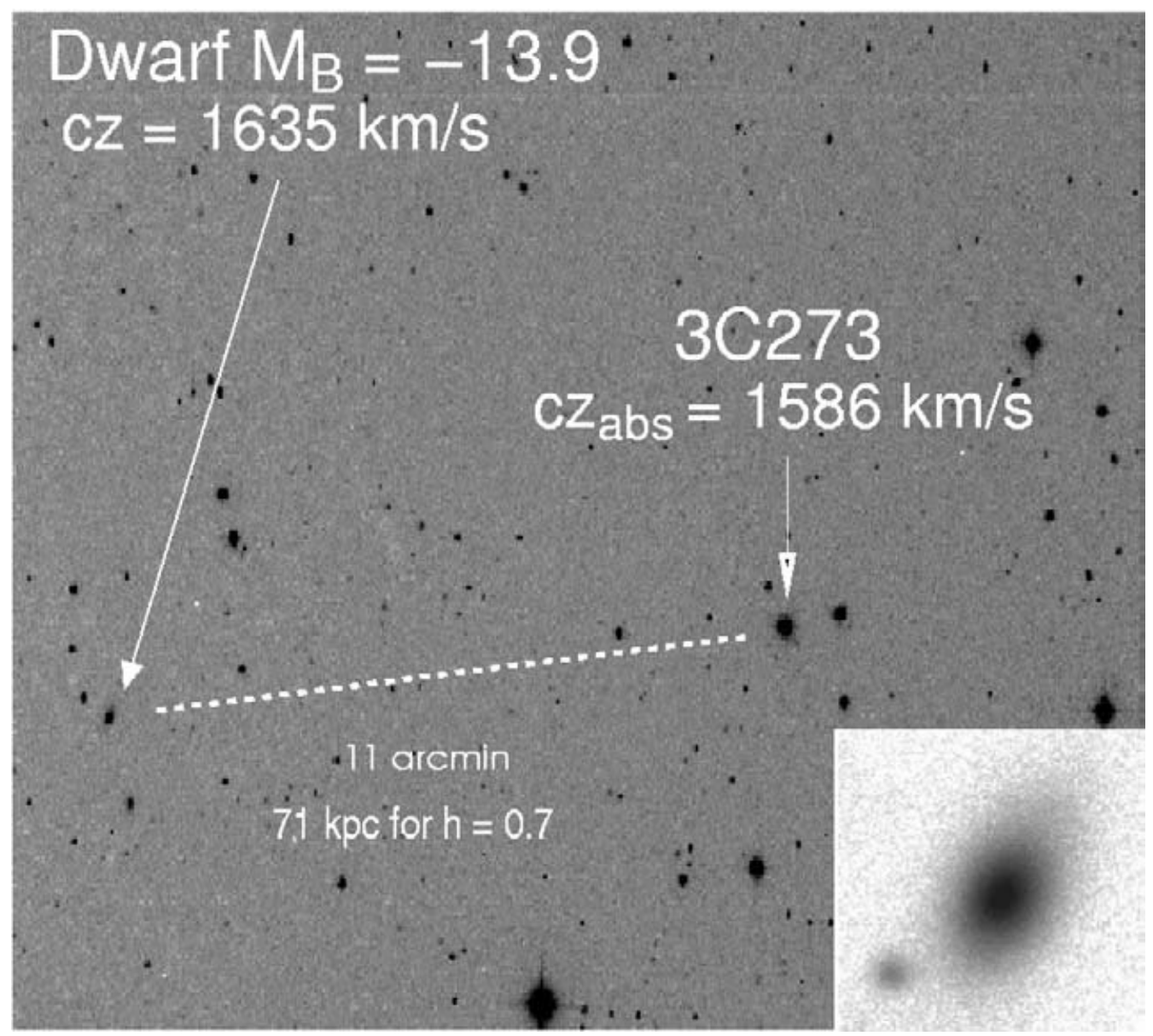

Figure 3. Digitized Sky Survey image of the region around 3C 273 (north up, east to left) showing the dwarf galaxy located $\sim 70 h_{70}^{-1} \mathrm{kpc}$ away from the absorber on the sky and at the same recession velocity to within the errors. The R-band image of the dwarf galaxy taken with the ARC $3.5 \mathrm{~m}$ telescope at Apache Point is shown as an insert at lower right. The surface brightness profile of this galaxy is well-fit by a pure exponential disk.

one massive starburst which ejected most or all of the gas from the galaxy to a distance of $\sim 100 h_{70}^{-1} \mathrm{kpc}$, this process would be sufficient to create several hundred weak metal-line absorbers per unit redshift. This number is comparable to the line density of all metalline absorbers at $z=2$. While this dwarf galaxy would have been much more luminous $\left(\mathrm{M}_{B} \approx-16.5\right)$ when it was "starbursting", the present day absence of gas means that it will no longer form stars and will eventually fade to the approximate luminosity of a Local Group dwarf spheroidal. Thus, the dwarf in Figure 3 is the expected intermediate stage between the "faint blue galaxies" seen at intermediate redshift and the dwarf spheroids (Babul \& Rees 1992). That such "Cheshire Cat" galaxies might be responsible for a large portion of the Ly $\alpha$ forest was predicted by Salpeter (1993) and Charlton (1995). Discovery of a similar dwarf near the RXJ $1230.8+01151666 \mathrm{~km} \mathrm{~s}^{-1}$ absorber would solidify this model. A galaxy survey of the RXJ $1230.8+0115$ region is now in progress. 


\section{Acknowledgements}

Financial support for "local" Ly $\alpha$ forest work at the University of Colorado comes from grants provided through HST GO program, \#s 6593, 8182, 8125, 9221 \& 9506, and NASA Theory Grant NAG5-7262. We thank Ray Weymann for the faint galaxy redshift survey work and his inspirational leadership in this field. We also thank Mark Giroux for photo- and collisional-ionization modeling and John Hibbard, Jacqueline van Gorkom and collaborators for the H I work at the VLA.

\section{References}

Babul, A. \& Rees, M.J. 1992, MNRAS, 255, 346

Bahcall, J.N. et al. 1991,ApJ, 377, L5

Bahcall, J.N. et al. 1993, ApJS, 87, 1

Cen, R. et al. 1994, ApJ, 427, L9

Charlton, J.C. 1995, in QSO Absorption Lines, ed. G. Meylan (Berlin:Springer) , 405

Cowie, L.L. \& Songaila, A. 1998, Nature, 394, 44

Davé, R., Hernquist, L., Katz, N. \& Weinberg, D.H. 1999, ApJ, 511, 521

Davé, R., et al. 2001, ApJ, 552, 473

Gottlöber, S., Lokas, E.L. Klypin, A. \& Hoffman, Y. 2003, MNRAS, 344, 715

Hu, E.M., Kim, T.-S., Cowie, L.L., Songaila, A. \& Rauch, M. 1995 AJ, 110, 1526

Huchra, J. P., et al. 1992 Bull. C.D.S., 41, 31 (2004 electronic version)

Jannuzi, B.T. et al. 1998, ApJS, 118, 1

McLin, K.M., Stocke, J.T., Weymann, R.J. \& Shull, J.M. 2001, ApJ, 574, L115

Morris, S.L., Weymann, R.J., Savage, B.D. \& Gilliland, R.L. 1991, ApJ, 377, L21

Morris, S.L., et al. 1993, ApJ, 419, 524

Penton, S.V., Stocke, J.T. \& Shull, J.M. 2000a, ApJS, 130, 121 (Paper I)

Penton, S.V., Shull, J.M. \& Stocke, J.T. 2000b, ApJ, 544, 150 (Paper II)

Penton, S.V., Stocke, J.T. \& Shull, J.M. 2002, ApJ, 565, 720 (Paper III)

Penton, S.V., Stocke, J.T. \& Shull, J.M. 2004, ApJS, in press (astro-ph/0401036; Paper IV)

Rosenberg, J.L., Ganguly, R., Giroux, M.L. \& Stocke, J.T. 2003, ApJ, 591, 677

Sargent, W.L.W. 1988, Proceedings of the QSO Absorption Line Meeting, ed. J.C . Blades, D. Turnshek \& C.A. Norman (Cambridge U. Press), 1

Salpeter, E.E. 1993, AJ, 106, 1265

Schaye, J. 2001, ApJ, 559, 507

Simcoe, R.A., Sargent, W.L.W. \& Rauch, M. 2004, ApJ, 606, 92

Stocke, J.T., Keeney, B.A., McLin, K.M., Rosenberg, J.L., Weymann, R.J. \& Giroux, M.L. 2004, ApJ, in press (astro-ph/0403042)

Tripp, T. M., et al. 2002 ApJ, 575, 697

van Gorkom, J.H., Bahcall, J.N., Jannuzi, B.T. \& Schneider, D.P., 1993, AJ, 106, 2213

Weymann, R.J. et al. 1998, ApJ, 506, 1 